


\section{Influência da adição de taninos elágicos na qualidade de vinhos merlot da região da Campanha}

\section{Influence of tannins ellagic addition in quality merlot wines of Campaign region}

\section{OPPEN ACCESS}

\section{Deisi Cerbaro ${ }^{1,(1 D}$}

César Valmor Rombaldi2, (iD

Ricardo Lemos Sainz ${ }^{1, *}$ (iD)

Gisele Alves Nobre ${ }^{1, \text { (iD }}$

1 Instituto Federal Sul Rio Grandense, Campus Pelotas Visconde da Graça, Brasil. Pelotas, RS, Brasil.

2 Universidade Federal de Pelotas, Faculdade de Agronomia Eliseu Maciel. Pelotas, RS, Brasil.

gialcantara9@hotmail.com

2 Empresa Brasileira de Pesquisa Agropecuária-EMBRAPA. Palmas, TO, Brasil.

*Autor para correspondência: ricardosainz@pelotas.ifsul.edu.br

\section{Informações adicionais}

Recebido em: 07/05/2016

Aceito em: $17 / 08 / 2016$

Publicado em: 30/09/2016

Editor:

Victor Hugo Gomes Sales

Instituto Federal do Amapá, Macapá, Amapá, Brasil. jbfs@ifap.edu.br

\section{Avaliação às cegas por pares}

Protocolos de revisão

Prot. $0982016 R 01$

Prot. $0982016 R 02$

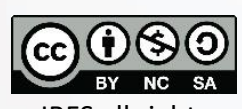

JBFS all rights

Copyright: (C) 2016
ID JBFS1032016

DOI 10.18067/jbfs.v3i3.103

\section{RESUMO}

No âmbito das técnicas de vinificação existem vários recursos disponíveis para potencializar a qualidade de vinhos. Frente às imensas possibilidades de combinações que podem resultar em vinhos de qualidade única este trabalho teve como objetivo avaliar o efeito e os benefícios da aplicação de taninos elágicos em substituição total ou parcial de anidrido sulfuroso nas propriedades químicas de vinhos da variedade Merlot. No experimento foram realizados os seguintes tratamentos: T1 (testemunha, vinificação tradicional com adição de metabissulfito de potássio $20 \mathrm{~g} \cdot \mathrm{hL}^{-1}$ ); T2 (vinificação tradicional sem adição de metabissulfito de potássio e sem adição de tanino); T3 (vinificação tradicional com adição de tanino elágico 30 g.hL ${ }^{-1}$ ); T4 (vinificação tradicional com adição de tanino elágico $30 \mathrm{~g} \cdot \mathrm{hL}^{-1}$ e metabissulfito de potássio $10 \mathrm{~g} \cdot \mathrm{hL}^{-1}$ ). Os tratamentos foram avaliados após a descuba, após o término da fermentação malolática e após o engarrafamento. Os resultados obtidos através deste trabalho indicam que é tecnicamente possível a produção de vinho tinto Merlot jovem sem a adição de $\mathrm{SO}_{2}$. Porém, mais estudos são necessários para otimização das técnicas de vinificação empregadas e a relação destas com os parâmetros avaliados.

Palavras-chave: Vinificação. Tintos. Maceração a frio, Tratamentos enológicos.

\section{ABSTRACT}

Within the winemaking techniques, there are several resources available to enhance the quality of wines. Faced with the immense possibilities of combinations that can result in unique quality wines this study aimed to evaluate the effects and benefits of the ellagic tannins application in full or partial replacement of sulfur dioxide in the wines from the variety Merlot chemical properties. In these experiments, the following treatments were performed: T1 (control, traditional vinification with potassium metabisulfite addition - 20 g.hL ${ }^{-1}$ ); T2 (traditional wine without the potassium metabisulfite addition and without tannin addition); T3 (traditional wine with the ellagic tannin addition - $30 \mathrm{~g} \cdot \mathrm{hL}^{-1}$ ); T4 (traditional wine with the addition of $30 \mathrm{~g} \cdot \mathrm{hL}^{-1}$ ellagic tannin and potassium metabisulfite $10 \mathrm{~g} \cdot \mathrm{hL}^{-1}$ ). The treatments were evaluated after the end of the maceration, after the malolactic fermentation and after bottling. The results obtained in this study indicate that it is technically possible to produce Merlot young red wine without the addition of $\mathrm{SO}_{2}$. But more studies are needed to optimize the employed winemaking techniques and their relationship to the parameters evaluated.

Keywords: Winemaking, Red wines, Cold maceration. Enological practices. 


\section{INTRODUÇÃO}

A qualidade dos vinhos brasileiros tem passado por constantes evoluções nos últimos anos, incorporando notáveis melhorias, principalmente no que diz respeito ao emprego de variedades Vitis vinifera e às diferentes técnicas enológicas. Tal condição permite classificá-los no quarto período de sua escala evolutiva, onde busca a afirmação da identidade regional. ${ }^{1}$ Assim, várias iniciativas e pesquisas têm sido desenvolvidas com o propósito de concretizar novas regiões vitivinícolas, por meio de técnicas que permitam melhor aproveitar as características de cada uma, obtendo vinhos de qualidade baseadas na originalidade.

No âmbito das técnicas de vinificação existem vários recursos disponíveis para potencializar a qualidade e a diferenciação de vinhos. A diminuição do uso de dióxido de enxofre tem sido uma tendência oriunda do mercado crescente de consumidores que buscam produtos com características diferenciadas, bem como vinhos orgânicos, agroecológicos e biodinâmicos. Boa parte das vinícolas se depara com essa demanda, havendo necessidade de se criarem alternativas de antioxidantes e produtos antimicrobianos alternativos, mantendo a qualidade dos vinhos. $^{2}$

A sulfitagem é amplamente e historicamente utilizada na vitivinicultura mundial, como meio de proteção a oxidação enzimática e química, e como seletora de micro-organismos, especialmente para leveduras vinárias. ${ }^{2}$ Entretanto, o uso do dióxido de enxofre deve ser racional, pois em doses elevadas pode retardar ou provocar paradas de fermentação, diminuir a intensidade de cor dos vinhos tintos e rosados, promover o surgimento de aromas desagradáveis. Ainda, tem sido associado a danos à saúde, especialmente processos alérgicos., Portanto, há uma tendência de buscar alternativas de consorciação, que viabilizem a redução das quantidades utilizadas deste aditivo em vinhos.

Atualmente há disponível alternativas de vinificação, como a adição de taninos enológicos no mosto, e manejos pré-fermentativos a frio, que modificando as condições de maceração, buscam melhores resultados que os esperados mediante o processo de maceração tradicional. ${ }^{5}$
O uso do tanino está autorizado com o objetivo de facilitar a precipitação de matérias protéicas em excesso e auxiliar nos processos de clarificação. Outros usos têm sido descritos, dadas as propriedades na melhoria do corpo, na eliminação de aromas e gostos atribuídos a fenômenos de redução, na estabilização da cor e na melhoria de aromas. ${ }^{6,7}$ Outra propriedade muito importante é sua capacidade anti-radical livre e de consumo de oxigênio diluído, propriedade interessante e de ampla exploração na área enológica. ${ }^{8}$

Assim sendo, o emprego de taninos enológicos poderia, em tese, ser um meio para a obtenção de vinhos com maior distinguibilidade, corrigindo, em parte, os problemas da matériaprima, com um perfil aromático mais amplo e definido, além de baixo conteúdo de substâncias tânicas não nobres (sem adstringência) e de maior estabilidade de compostos antociânicos. ${ }^{9}$ Os taninos participam da cor e do gosto da uva e do vinho. Conferem "corpo" ao vinho, além de serem diretamente responsáveis pelas sensações sensoriais de adstringência e de amargor. São compostos fenólicos caracterizados pela capacidade de combinar-se com as proteínas e outros polímeros como os polissacarídeos. Isso explica sua adstringência, causada pela precipitação de proteínas e de glicoproteínas da saliva, gerando uma sensação de secura na boca, que se denomina adstringência, $^{10}$ sensações tátil de grande importância na enologia. ${ }^{11}$

Quimicamente, os taninos são moléculas fenólicas relativamente volumosas, resultantes da polimerização de moléculas elementares de função fenol. Sua configuração espacial está relacionada com sua reatividade. Segundo a natureza das moléculas elementares, diferenciam-se em taninos hidrolisáveis ou gálicos e taninos condensados ou catéquicos. Os taninos hidrolisáveis são naturais da uva, presentes na casca e sementes, enquanto os condensados são encontrados na madeira de carvalho. $^{12}$

Os taninos hidrolisáveis também são denominados galotaninos e elagitaninos, por liberarem ao meio maior quantidade de ácido gálico ou ácido elágico, após hidrólise ácida. Na madeira de carvalho encontram-se, principalmente, dois elagitaninos isômeros, a vescalagina e a castalagina. ${ }^{13} \mathrm{O}$ tanino elágico apresenta fácil oxidação em meio 
hidroalcoólico e propriedades gustativas agradáveis, que são transferidas ao vinho durante seu envelhecimento em barricas de carvalho. ${ }^{12}$

Quando taninos reagem com as antocianinas, formam substâncias coloridas quimicamente estáveis. ${ }^{14}$ São os compostos que mais importância tem no que diz respeito à cor das uvas e dos vinhos. As antocianinas representam parte importante, quantitativamente e qualitativamente dos flavonóides, das uvas das variedades tintas. ${ }^{15} \mathrm{O}$ uso de taninos exógenos na indústria vinícola vem ganhando impulso, com um número expressivo de empresas fazendo uso dos mesmos, sem que se tenham maiores estudos validando a contribuição na qualidade do vinho. ${ }^{16}$ Segundo Alvarez ${ }^{17}$, os principais taninos utilizados na enologia são o elágico, o gálico e o proantocianidinico.

O tanino para uso enológico está definido pelo CODEX Enológico Internacional da Organização Mundial da Uva e do Vinho (OIV), através da resolução Oeno $12 / 2002,{ }^{18}$ onde é permitido para utilização como clarificante, devido às suas propriedades reativas com proteínas. Os taninos enológicos são formulações comerciais de taninos extraídos das mais diversas fontes, incluindo os de origem própria da uva, ou a partir de partes lignificadas das plantas, de frutos, e de protuberâncias produzidas por agentes patógenos. Até então, os taninos mais utilizados em enologia provêm de diferentes espécies vegetais, notadamente de várias espécies de carvalho (Quercus sp.), de castanheira (Castanea sativa Mill.), de quebracho (Schinopsis sp.), de mirabolano (Terminalia chebula) e outras ${ }^{10,19,20}$ Os taninos elágicos são obtidos principalmente a partir da casca e troncos de árvores como o carvalho (Quersus $s p$ ) de diferentes variedades. ${ }^{21}$

Os taninos elágicos podem ajudar a proteger as antocianinas da oxidação, uma vez que eles são capazes de atuar regulando fenômenos redox, ${ }^{22}$ favorecendo a condensação entre antocianinas e taninos com acetaldeído e formando complexos moleculares. ${ }^{23}$ Permitem assim, uma maior estabilização da matéria corante, protegendo as antocianinas e os próprios taninos endógenos, conservando a cor dos vinhos. Outro aspecto a considerar é a possibilidade de que estes taninos contribuem para aumentar a cor do vinho através do fenômeno de copigmentação. ${ }^{7} \mathrm{O}$ efeito da adição de taninos enológicos sobre o corpo e estrutura do vinho parece ser evidente, já que em boa parte dos casos, é observado um aumento do índice de polifenóis totais, assim como incremento nas sensações de corpo e estrutura. ${ }^{24}$ Os taninos condensados e os taninos elágicos tem-se mostrado melhor efeito para este fim. ${ }^{22}$ Porém, quando utilizado em excesso, reporta ao vinho dureza e sabores amargos. ${ }^{25}$

As uvas da casta Merlot são originárias da região de Bordeaux, mais precisamente de Saint-Émillion, sendo uma das principais uvas responsáveis pelos vinhos tintos dessa região. No Rio Grande do Sul, ela foi introduzida através da Estação Agronômica de Porto Alegre, de onde foi difundida para a Serra Gaúcha, mais intensamente a partir da década de 1970, marcando o início da produção de vinhos finos varietais brasileiros, juntamente com outras variedades de Vitis vinifera. O vinho Merlot apresenta aspecto bom devido, principalmente, à coloração vermelho-violáceo. Quanto ao olfato, não apresenta aroma pronunciado típico. Gustativamente, ele impressiona pelo equilíbrio e maciez. ${ }^{26} \mathrm{O}$ vinho Merlot apresenta aspecto bom devido, principalmente, à coloração vermelho-violáceo. Quanto ao olfato, não apresenta aroma pronunciado típico. Gustativamente, ele impressiona pelo equilíbrio e maciez. ${ }^{23}$

Apresenta grande potencial e vem se destacando, sendo a segunda vinífera tinta mais plantada no mundo, apresentando boa produção de uvas e elevado potencial enológico, resultando na elaboração e obtenção de vinhos com qualidade e tipicidade. ${ }^{27} \mathrm{~A}$ qualidade das uvas e vinhos Merlot produzidas na região da Campanha do RS é um dos grandes destaques enológicos deste terroir. A ampla distribuição e produção da variedade aliada a sua qualidade na região de estudo fundamenta a escolha da variedade Merlot para este estudo.

Este trabalho teve como objetivo avaliar o efeito e os benefícios da aplicação de taninos elágicos em substituição total ou parcial de anidrido sulfuroso nas propriedades químicas de vinhos da variedade Merlot, produzidas na região da Campanha Gaúcha (RS).

\section{MATERIAL E MÉTODOS}

\section{a) Matéria Prima}

Foram utilizadas uvas da variedade Merlot (Vitis vinifera), safra 2013, provenientes de vinhedo comercial de 8 anos de idade, localizado em Bagé, Região da Campanha Gaúcha, Rio Grande do Sul. O 
vinhedo com exposição norte-sul foi conduzido em cordão esporonado, no sistema espaldeira, portaenxerto Paulsen 1103, e com espaçamento 1,5 metros entre plantas e 3 metros entre linhas, mantendo uma produção máxima de 10 toneladas por hectare. A colheita foi realizada com $19^{\circ}$ Babo e excelente estado sanitário. As microvinificações, em triplicatas, foram realizadas na Sala de Microvinificação no Instituto Federal Sul-riograndense Campus Pelotas - Visconde da Graça.

\section{b) Delineamento Experimental}

A uva foi vinificada conforme descrito no experimento a seguir. Após a fermentação alcoólica os vinhos foram acondicionados em vasilhames de 5 litros com sistema de batoque hidráulico, para fins de fermentação malolática, sendo monitorada por cromatografia de papel (Figura 1 e 2).

A estabilização a frio aconteceu 8 meses após a vinificação, sendo realizada em câmara fria a $0^{\circ} \mathrm{C}$ por 4 semanas. Concluída esta etapa, o vinho foi engarrafado. Os Taninos Elágicos (Ellagitan La ${ }^{\circledR}$ ) utilizados foram na forma líquida solúvel, conforme recomendação do fabricante na dosagem de 30 g.hL ${ }^{-1}$.

\section{c) Procedimento Experimental}

Após a colheita, a uva foi processada, passando por desengace, esmagamento, adição de complexo enzimático na dosagem de 2 g.hL ${ }^{-1}$ (Endozym Rouge Liquid ${ }^{\circ}$ ) a partir de uma solução comercial concentrada e leveduras seca ativa na dosagem de $25{\mathrm{~g} . h L^{-1}}$ (Zymazil $^{\circ}$ ). O material foi acondicionado em recipientes de vidro de 20 litros em ambiente com temperatura controlada de 20 a $25^{\circ} \mathrm{C}$ para proceder com a fermentação alcoólica. Durante a maceração foram realizadas três remontagens diárias, por 5 dias, até a descuba, utilizando-se somente o vinho flor. A aplicação dos tratamentos se deu da seguinte forma:

- Tratamento 1: Testemunha, vinificação tradicional com adição de metabissulfito de potássio a $20 \mathrm{~g} \cdot \mathrm{hL}^{-1}$;

- Tratamento 2: Vinificação tradicional sem adição de metabissulfito de potássio e sem adição de Tanino;

- Tratamento 3: Vinificação tradicional com adição de Tanino Elágico a 30g.hL ${ }^{-1}$;
- Tratamento 4: Vinificação tradicional com adição de Tanino Elágico a $30 \mathrm{~g} \cdot \mathrm{hL}^{-1} \mathrm{e}$ metabissulfito de potássio a $10 \mathrm{~g} \cdot \mathrm{hL}^{-1}$.

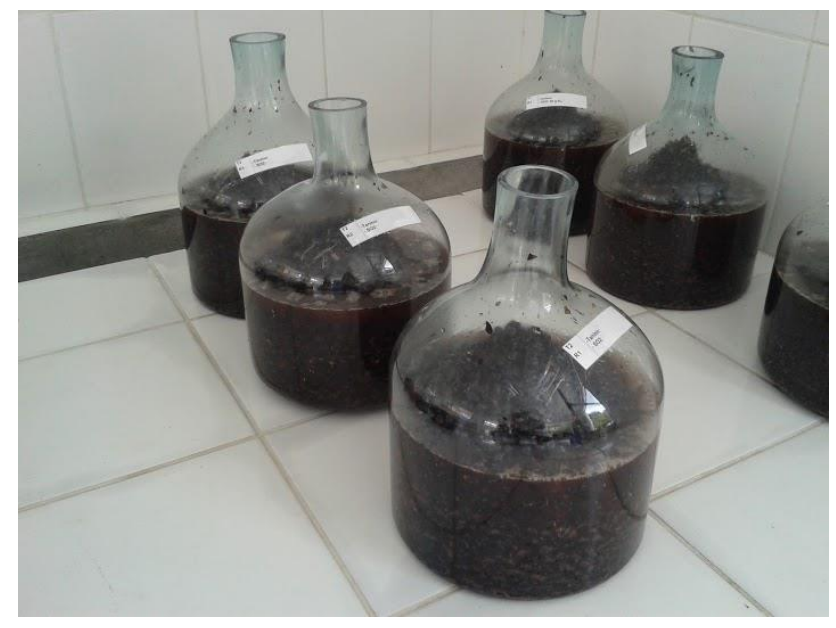

Figura 1. Acondicionamento da uva em garrafões de 20 litros após desengace.

Fonte: CERBARO, 2013.

Figure 1.Grape Packaging in carboy of 20 liters after destemming.

Source: CERBARO, 2013.

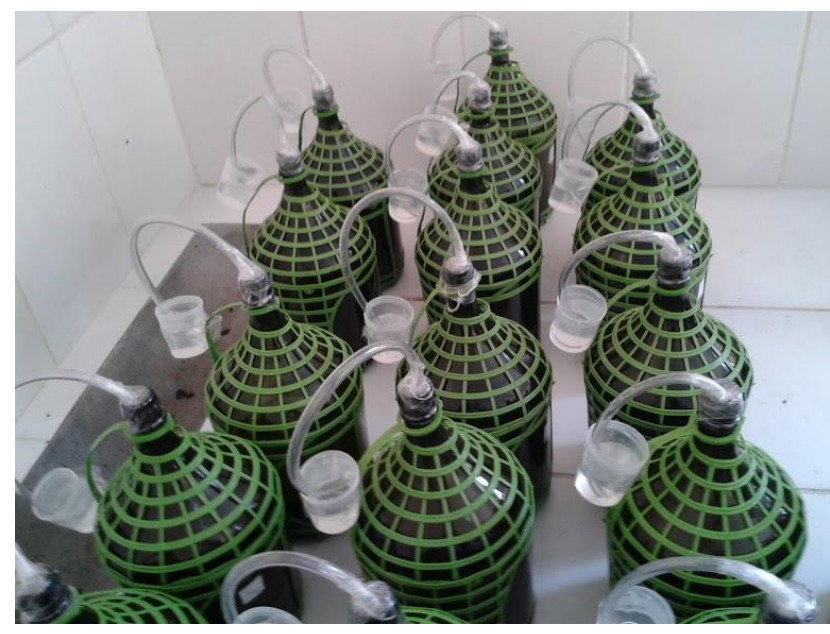

Figura 2, Acondicionamento do vinho após fermentação alcoólica, com sistema de batoque hidráulico, para conclusão da fermentação malolática.

Fonte: CERBARO, 2013.

Figure 2. Wine Packaging after alcoholic fermentation, with hydraulic bung system, to complete the malolactic fermentation.

Source: CERBARO, 2013

\section{d) Avaliações}

As variáveis foram analisadas nos laboratórios do Departamento de Ciência e Tecnologia de Alimentos da Universidade Federal de Pelotas, Laboratório de Análises Físico-químicas 
de Alimentos do Campus Pelotas - Visconde da Graça do IFSUL e, no Laboratório de Controle Analítico da Vinícola Campestre Ltda.

As avaliações de $\mathrm{SO}_{2}$ livre, $\mathrm{SO}_{2}$ total, acidez total, teor alcoólico, $\mathrm{pH}$, índice de cor e tonalidade aconteceram logo após a descuba, após a fermentação malolática e após o engarrafamento das amostras. O índice de polifenóis totais, de antocianinas totais e atividade antioxidante foram avaliadas após a fermentação malolática e após o engarrafamento, e acidez volátil após o engarrafamento.

\section{e) Análises Físico-químicas}

As análises físico-químicas de teor alcoólico (\% v/v), $\mathrm{pH}$, acidez total (meq. $\left.\mathrm{L}^{-1}\right)$, acidez volátil (meq. $\left.\mathrm{L}^{-1}\right), \mathrm{SO}_{2}$ total $\left(\mathrm{mg}^{-\mathrm{L}^{-1}}\right)$ e $\mathrm{SO}_{2}$ livre $\left(\mathrm{mg} . \mathrm{L}^{-1}\right.$ ) foram determinadas conforme descrito por Rizzon ${ }^{28}$

\section{f) Índice de Cor}

Caracterizados pela medida da absorbância a $420 \mathrm{~nm}, 520 \mathrm{~nm}$ e $620 \mathrm{~nm}$ em espectrofotômetro (UV/VIS Jenway 6700). O índice de cor se deu pela soma do resultado das três absorbâncias, conforme descrito por Ribéreau-Gayon et al. ${ }^{29}$

\section{g) Tonalidade}

A tonalidade foi determinada conforme descrito por Ribéreau-Gayon et al., ${ }^{29}$ através da razão entre os resultados das absorbâncias de 420 $\mathrm{nm}$ e $520 \mathrm{~nm}$, sendo os resultados expressados em índice.

\section{h) Índice de Polifenóis Totais}

O índice de polifenóis totais foi determinado pelo método espectrofotométrico em $280 \mathrm{~nm}$ de acordo com o protocolo de Ribéreau-Gayon et al., ${ }^{29}$ e os resultados são expressos em índice.

\section{i) Fenóis Totais}

O conteúdo de fenólicos totais de acordo com o método de Singleton e Rossi, ${ }^{30}$ em espectrofotômetro (UV/VIS Jenway 6700), utilizando-se ácido gálico como padrão, com leituras da absorbância no comprimento de onda de $765 \mathrm{~nm}$. Os resultados obtidos foram expressos em mg. $\mathrm{L}^{-1}$ de ácido gálico.

\section{j) Antocianinas Totais}

A avaliação do teor de antocianinas totais foi realizada por método espectrofotométrico, de acordo com o protocolo de Lees e Francis, ${ }^{32}$ utilizando-se etanol acidificado. As medidas foram realizadas a $520 \mathrm{~nm}$ em espectrofotômetro (UV/VIS Jenway 6700) e os resultados foram expressos em $\mathrm{mg} .100 \mathrm{~mL}^{-1}$ de cianidina 3-glicosídio.

\section{k) Atividade Antioxidante}

A atividade antioxidante foi avaliada utilizando-se o método do sequestro de radicais livres do DPPH (2,2-difenil-1-picrilhidrazila) com adaptações de Brand-Williams et al., ${ }^{33}$.As medidas foram realizadas a $517 \mathrm{~nm}$ em espectrofotômetro (UV/VIS Jenway 6700) no tempo padrão de leitura de 60 minutos após a adição da amostra; a reação foi realizada no escuro e em temperatura média de $25^{\circ} \mathrm{C}$. O tempo de reação foi definido com base em testes preliminares realizados anteriormente. Os resultados foram expressos em porcentagem de inibição do radical DPPH.

\section{I) Análise Estatística}

Os resultados foram avaliados através da análise de variância ANOVA e pelo teste de Tukey, ambos a $5 \%$ de significância, utilizando-se do Programa Statistica $7.0^{\circ}$.

\section{RESULTADOS E DISCUSSÃO}

\section{a) Características Físico-Químicas}

Os resultados das análises físico-químicas de teor alcoólico, $\mathrm{pH}$, acidez total, acidez volátil, $\mathrm{SO}_{2}$ total e $\mathrm{SO}_{2}$ livre estão apresentados na Tabela 1 , a seguir. O teor alcoólico situou-se entre 11,88 e $12,29 \%(\mathrm{v} / \mathrm{v})$. Os valores de $\mathrm{pH}$ não foram influenciados pelos tratamentos no momento do engarrafamento.

A acidez total ficou compreendida entre 71,06 e 102,70 meq. L $^{-1}$, o que representa aproximadamente 5,33 a 7,70 g. L $^{-1}$ de ácido tartárico. No experimento, considerado o tratamento testemunha, foi observada diferença significativa, com valores inferiores aos demais, em todos os momentos de avaliação, após a descuba (D), após fermentação malolática (F) e após o engarrafamento $(\mathrm{E})$.

Os resultados de acidez volátil encontrados, não diferiram estatisticamente entre os tratamentos abordados no experimento.

$\mathrm{O} \mathrm{SO}_{2}$ total ficou compreendido entre $10,76 \mathrm{e}$ 19,06 meq. $L^{-1}$ no momento do engarrafamento, sendo 
Tabela 1. Resultados analíticos das características físico-químicas de $\mathrm{pH}$, acidez total (meq. $\left.\mathrm{L}^{-1}\right)$, acidez volátil (meq. $\left.\mathrm{L}^{-1}\right)$, teor alcoólico (\% v/v), $\mathrm{SO}_{2}$ total $\left(\mathrm{mg} . \mathrm{L}^{-1}\right)$ e $\mathrm{SO}_{2}$ livre $\left(\mathrm{mg} \cdot \mathrm{L}^{-1}\right)$, de vinhos Merlot tratados com diferentes dosagens de taninos elágicos e $\mathrm{SO}_{2}$ avaliados após a descuba (D), após fermentação malolática (F) e após o engarrafamento (E).

Table 1. Analytical results of the physico-chemical characteristics of $\mathrm{pH}$, total acidity $\left(\mathrm{mEq} \cdot \mathrm{L}^{-1}\right)$, volatile acidity $\left(m E q . L^{-1}\right)$, alcohol $(\% \mathrm{v} / \mathrm{v})$, Total SO2 $\left(\mathrm{mg} \mathrm{L}^{-1}\right)$ and SO2 free $\left(\mathrm{mg}^{\mathrm{L}} \mathrm{L}^{-1}\right)$, Merlot wine treated with different dosages of ellagic tannins and SO2 evaluated after descuba (D) after malolactic fermentation (F) and after bottling (E).

\begin{tabular}{|c|c|c|c|c|c|}
\hline Avaliação & & $\mathrm{T} 1$ & $\mathrm{~T} 2$ & T3 & $\mathrm{T} 4$ \\
\hline & $\mathrm{D}$ & $10,93 c$ & $11,66 b$ & $12,26 a$ & $12,00 a b$ \\
\hline \multirow[t]{3}{*}{ Teor Alcoólico } & $\mathrm{F}$ & $12,53 a$ & $13,00 a$ & $13,00 a$ & $13,00 a$ \\
\hline & $E$ & $11,88 a$ & $12,29 a$ & $11,98 a$ & $11,88 a$ \\
\hline & $\mathrm{D}$ & $3,44 a$ & $3,43 a$ & $3,41 a$ & $3,41 a$ \\
\hline \multirow[t]{3}{*}{$\mathrm{pH}$} & $\mathrm{F}$ & $3,73 a$ & $3,70 a$ & $3,67 a$ & $3,69 a$ \\
\hline & $E$ & $3,67 a$ & $3,67 a$ & $3,66 a$ & $3,66 a$ \\
\hline & $\mathrm{D}$ & $76,65 b$ & $86,60 a$ & $87,35 a$ & $88,65 a$ \\
\hline \multirow[t]{2}{*}{ Acidez Total } & $\mathrm{F}$ & $83,25 b$ & $88,65 a$ & $90,70 a$ & $88,00 a$ \\
\hline & $E$ & $71,06 b$ & $79,46 a$ & $83,06 a$ & $79,20 a$ \\
\hline \multirow[t]{2}{*}{ Acidez Volátil } & $\mathrm{E}$ & $5,86 a$ & $3,73 a$ & $4,00 a$ & $6,80 a$ \\
\hline & $\mathrm{D}$ & $54,66 a$ & $16,80 \mathrm{~b}$ & $17,70 b$ & $17,06 b$ \\
\hline \multirow[t]{3}{*}{$\mathrm{SO}_{2}$ Total } & $F$ & $35,60 a$ & $25,60 a$ & $25,06 a$ & $38,93 a$ \\
\hline & $E$ & $10,76 b$ & $13,13 a$ & $12,50 a$ & $12,66 a$ \\
\hline & D & $16,00 a$ & $9,86 a b$ & $9,06 a b$ & $8,53 b$ \\
\hline \multirow[t]{2}{*}{$\mathrm{SO}_{2}$ Livre } & $\mathrm{F}$ & $18,66 a$ & $13,33 b$ & $16,53 a b$ & $19,73 a$ \\
\hline & $E$ & $3,53 a$ & $4,10 a$ & $3,93 a$ & $5,23 a$ \\
\hline
\end{tabular}

T1: Testemunha $\mathrm{SO}_{2}$ de maneira tradicional $\left(20 \mathrm{~g} \cdot \mathrm{hL}^{-1}\right)$; $\mathrm{T} 2$ : Sem adição de tanino e $\mathrm{SO}_{2} ; \mathrm{T} 3$ : 30 g.hL $\mathrm{hL}^{-1}$ de tanino elágico; T4: $30 \mathrm{~g} \cdot \mathrm{hL}^{-1}$ de tanino elágico $+10 \mathrm{~g} \cdot \mathrm{hL}^{-1}$ de $\mathrm{SO}_{2}$.

* Letras distintas na mesma linha indicam diferenças significativas $(p \leq 0,05)$ pelo teste de Tukey.

T1: Witness SO2 traditional way (20 g.hL-1); T2: No addition of tannin and SO2; T3: $30 \mathrm{~g} \cdot \mathrm{hL}^{-1}$ ellagic tannin; T4: $30 \mathrm{~g} \cdot \mathrm{hL}^{-1}$ ellagic tannin $+10 \mathrm{~g} \cdot \mathrm{hL} \mathrm{SO} 2^{-1}$.

* Different letters in the same line indicate significant differences $(p \leq 0.05)$ by Tukey test.

que os tratamentos testemunhas (T1) do experimento tiveram valores inferiores aos demais.

Os valores de $\mathrm{SO}_{2}$ livre encontrados no experimento tiveram variações nas avaliações após a descuba (D) e após a fermentação malolática (F), não diferindo estatisticamente no momento do engarrafamento. b) Características Polifenólicas

Os resultados das análises da matriz polifenólica de índice de cor, tonalidade, índice de polifenóis totais, fenóis totais, antocianinas totais e atividade antioxidante estão apresentados na Tabela 2, a seguir. 
Tabela 2. Resultados das análises da matriz polifenólica de índice de cor, tonalidade, índice de polifenóis totais (IPT), fenóis totais ( $\mathrm{mg} \cdot \mathrm{L}^{-1}$ de ácido gálico), antocianinas totais $\left(\mathrm{mg} .100 \mathrm{~mL}^{-1} \mathrm{de}\right.$ cianidina 3-glicosídio) e atividade antioxidante (\% de inibição do radical DPPH) de vinhos Merlot tratados com diferentes dosagens de taninos elágicos e $\mathrm{SO}_{2}$ avaliados após a descuba (D), após fermentação malolática (F) e após o engarrafamento (E).

Table 2. Results of the analysis of polyphenolic matrix color index, hue, total polyphenol index (TPI), total phenolics (mg L-1 gallic acid), anthocyanins ( $m g .100 \mathrm{~mL}^{-1}$ cyanidin 3- glucoside) and antioxidant activity (\% inhibition of DPPH) Merlot wine treated with different dosages of ellagic tannins and $\mathrm{SO} 2$ evaluated after descuba (D) after malolactic fermentation (F) and after bottling (E).

\begin{tabular}{llllll}
\hline \multicolumn{1}{l}{ Avaliação } & & T1 & T2 & T3 & T4 \\
\hline \multirow{2}{*}{ Índice de Cor } & D & $0,740 a$ & $0,846 a$ & $0,850 a$ & 0,841 a \\
& F & $0,921 a$ & $0,870 a$ & $0,852 a$ & 1,012 a \\
& E & $0,949 a$ & $0,847 a$ & $0,859 a$ & $0,843 a$ \\
\hline \multirow{2}{*}{ Tonalidade } & D & $0,597 a$ & $0,577 a$ & $0,600 a$ & $0,604 a$ \\
& F & $0,723 a$ & $0,778 a$ & $0,768 a$ & $0,775 a$ \\
IPT & E & $0,742 \mathrm{~b}$ & $0,820 a$ & $0,810 a$ & $0,834 a$ \\
\hline \multirow{2}{*}{ Fenóis Totais } & F & $22,50 a$ & $21,30 a$ & $22,73 a$ & $20,50 a$ \\
& E & $31,03 a$ & $31,46 a$ & $32,16 a$ & $31,46 a$ \\
\hline \multirow{2}{*}{ Antocianinas Totais } & F & $16,66 a$ & $17,16 a$ & $17,27 a$ & $16,89 a$ \\
\hline Atividade & E & $17,01 a$ & $16,94 a$ & $17,56 a$ & $17,25 a$ \\
\hline Antioxidante & F & $58,35 a$ & $61,88 a$ & $60,55 a$ & $57,63 a$ \\
\hline
\end{tabular}

T1: Testemunha $\mathrm{SO}_{2}$ de maneira tradicional $\left(20 \mathrm{~g} \cdot \mathrm{hL}^{-1}\right) ; \mathrm{T} 2$ : Sem adição de tanino e $\mathrm{SO}_{2}$; T3: $30 \mathrm{~g} \cdot \mathrm{hL}^{-1}$ de tanino elágico; T4: $30 \mathrm{~g} \cdot \mathrm{hL}^{-1}$ de tanino elágico $+10 \mathrm{~g} \cdot \mathrm{hL}^{-1}$ de $\mathrm{SO}_{2}$.

* Letras distintas na mesma linha indicam diferenças significativas $(p \leq 0,05)$ pelo teste de Tukey.

T1: Witness SO2 traditional way (20 g.hL $\left.{ }^{-1}\right) ;$ T2: No addition of tannin and SO2; T3: $30 \mathrm{~g} \cdot \mathrm{hL}^{-1}$ ellagic tannin; T4: $30 \mathrm{~g} \cdot \mathrm{hL}^{-1}$ ellagic tannin $+10 \mathrm{~g} \cdot \mathrm{hL} \mathrm{SO2-}{ }^{1}$.

* Different letters in the same line indicate significant differences $(p \leq 0.05)$ by Tukey test.

Os índices de cor encontrados neste trabalho variaram entre 0,740 e 1,012 . Os tratamentos do experimento não sofreram influência. Quando avaliados após a fermentação malolática (F), e após engarrafamento (E), o que simboliza o vinho estável e pronto para o consumo, os valores foram significativamente iguais.

Os valores de tonalidade encontrados variaram entre 0,742 e 0,834 no momento do engarrafamento. No experimento 1 após o engarrafamento todos os tratamentos tiveram valores superiores à testemunha, diferindo estatisticamente.

No experimento o índice de polifenóis totais dos tratamentos não diferiram estatisticamente. Após o engarrafamento os tratamentos não diferiram. Os tratamentos não afetaram significativamente os teores de fenóis totais e 
antocianinas totais no experimento. Os valores de fenóis totais variaram entre 16,66 e 18,22 mg. $\mathrm{L}^{-1}$ de ácido gálico. Os resultados de antocianinas totais ficaram compreendidos entre 57,63 a 63,88 mg.100 $\mathrm{mL}^{-1}$ de cianidina 3-glicosídio após a fermentação malolática (F), e 23,28 a $27,01 \mathrm{mg} .100 \mathrm{~mL}^{-1}$ de cianidina 3-glicosídio quando avaliados após o engarrafamento $(E)$.

A atividade antioxidante do tratamento com uso de tanino elágico e $\mathrm{SO}_{2}$ (T4) do experimento, foi superior aos demais no momento do engarrafamento. Quando avaliados pós a fermentação malolática $(F)$, os tratamentos não mostraram influência em nenhum dos experimentos.

\section{Discussões}

\section{a) Características Físico-Químicas}

Os resultados das análises físico-químicas encontram-se dentro dos Padrões de Identidade e Qualidade estabelecidos pela Legislação Brasileira, como mostram as Tabelas 1 e 2.

$O$ etanol provém essencialmente da fermentação alcoólica do açúcar presente no mosto. $\mathrm{O}$ etanol tem efeito sobre a estabilidade e o gosto do vinho. Altos teores de álcool etílico no vinho determinam maior poder antisséptico, proporcionando maior longevidade e conservação. Os valores de teor alcoólico encontrados no momento do engarrafamento demonstram o potencial enológico das uvas provenientes da Região da Campanha. Os tratamentos que foram expostos a manejos pré-fermentativos a frio tiveram teores alcoólicos superiores à testemunha. Favre, Charamelo e González-Neves, ${ }^{34}$ encontraram resultados similares entre seus tratamentos, quando trabalharam com maceração préfermentativa a frio e uso de taninos exógenos em vinhos da variedade Tannat.

Os valores de $\mathrm{pH}$ encontram-se normalmente entre 3 e 4 . Valores mais baixos conferem uma maior estabilidade microbiológica e físico-química, e aumenta a fração antisséptica do dióxido de enxofre. ${ }^{29}$ Vinhos com valores de $\mathrm{pH}$ mais altos representam uma menor força ácida, sendo mais suscetíveis às alterações oxidativas e biológicas, podendo resultar em um produto de baixa conservação.
Pode-se considerar que os valores de $\mathrm{pH}$ $(3,33$ a 3,77$)$ encontrados neste trabalho são medianos e estão dentro de uma faixa de segurança quanto a estabilidade microbiológica. Também estão de acordo com Miele, Rizzon e Mandelli ${ }^{35} \mathrm{em}$ estudo sobre efeito de diferentes manejos do dossel vegetativo da videira e seu efeito na composição do vinho Merlot.

$A$ acidez total de um mosto ou vinho leva em consideração todos os tipos de ácidos, tanto os ácidos minerais como ácido fosfórico, os ácidos orgânicos e também aminoácido, cuja contribuição para a acidez na titulação ainda não é bem conhecida. ${ }^{29}$ A Legislação Brasileira (Lei no 10970 de $12 / 11 / 2004$ ) permite valores de acidez total de 55 a 130 meq. L $^{-1}$. Os resultados analíticos encontrados estão dentro da faixa esperada para vinhos brasileiros e vão de acordo com os encontrados por Rizzon e Mielle ${ }^{36}$ em trabalhos de caracterização de vinhos Merlot elaborados com uvas provenientes da Serra Gaúcha, bem como em trabalhos realizados por Rizzon, Zanus e Mielle ${ }^{37}$ avaliando três regiões vitivinícolas do Rio Grande do Sul.

A acidez volátil está diretamente ligada à qualidade de um vinho, e todos os valores apresentaram-se abaixo de 20 meq. $\mathrm{L}^{-1}$, que é o valor máximo permitido pela legislação brasileira. ${ }^{38}$ Esses resultados mostram que mesmo sem uso ou reduzida quantidade de dióxido de enxofre, os vinhos não sofreram alteração microbiológica no que refere ao parâmetro analítico de acidez volátil. Segundo Rodrígues, ${ }^{39}$ os taninos enológicos servem como antioxidantes naturais, reforçando a ação protetora do anidrido sulfuroso e mantendo a sua fração livre por mais tempo, em comparação com vinhos onde não foram adicionados taninos exógenos.

Os agentes sulfitantes são classificados como aditivos alimentares e atuam na inibição da deterioração provocada por bactérias, fungos e leveduras em alimentos ácidos, e na inibição de reações de escurecimento enzimático e não enzimático durante processamento e estocagem. Adicionalmente, os sulfitos são utilizados como agentes antioxidantes e redutores em várias aplicações tecnológicas..$^{40-42}$ É importante ressaltar que o $\mathrm{SO}_{2}$ contido no vinho está presente em duas formas: livre e combinado.

Os dados analíticos encontrados para $\mathrm{SO}_{2}$ total revelam a presença de dióxido de enxofre em 
todos os tratamentos, mesmo quando seu uso foi restringido. $\mathrm{O}$ tratamento $\mathrm{T} 2$ (maceração préfermentativa a frio e $30 \mathrm{~g} \cdot \mathrm{hL}^{-1}$ de tanino elágico) obteve valor estatisticamente superior. Já é de senso comum que algumas cepas de leveduras têm capacidade de produzir compostos de enxofre, ${ }^{43,44}$ bem como as bactérias láticas no decorrer da fermentação malolática. ${ }^{45,46}$ Este fato pode justificar os valores de $\mathrm{SO}_{2}$ livre e $\mathrm{SO}_{2}$ total encontrados nos vinhos de todos os tratamentos.

Também, é importante destacar que, como pode ser verificado na tabela 1 , com exceção do T1 após a descubra e T4 após a fermentação malolática, em todos os demais tratamentos e em todas as etapas do processo o teor de $\mathrm{SO}_{2}$ total foi cerca de 10 vezes inferior ao limite estabelecido pela legislação. E, mesmo nos tratamentos T1 após a descubra e T4 após a fermentação malolática, o teor de $\mathrm{SO}_{2}$ foi bem inferior ao estabelecido pela legislação.

\section{b) Características polifenólicas}

Crespy $^{20}$ afirma que os taninos contribuem de uma forma geral, sendo que os taninos elágicos acentuam a cor escura dos vinhos, formando combinações estáveis com as antocianinas ou mesmo, para o aumento temporário da copigmentação. De acordo com estas informações, Côrte-Real ${ }^{47}$ explica que os taninos elágicos têm capacidade de reagir com o oxigênio apresentando várias funções hidroxi $(\mathrm{OH})$ em posição orto, conferindo-lhes a propriedade de atuar como antioxidantes. Estes fenômenos têm como efeito a redução dos riscos da perda de matéria corante.

No momento da descuba, o tratamento testemunha do primeiro experimento apresentou menores médias, ainda que sem diferença estatística. Isso sugere que os taninos exógenos podem ter influenciado esta variável, concordando com vários autores que já estudaram essa temática ${ }^{8,9,47}$

Os índices de cor encontrados neste trabalho variaram entre 0,740 e 1,012 . Zocche ${ }^{48}$ encontrou valores de 0,811 para variedade Merlot da Região da Campanha quando considerado maturação industrial. Rizzon e Miele, ${ }^{36}$ em estudo de caracterização de vinhos Merlot provenientes da Serra Gaúcha encontraram valores de 0,655, inferiores aos encontrados neste experimento.
A tonalidade é indicativa do desenvolvimento da cor para tons laranja, sendo mais baixa em vinhos novos do que em vinhos envelhecidos. Vinhos apresentando uma tonalidade igual ou inferior a 0,6 são considerados vinhos novos com um tempo de maturação relativamente curto. ${ }^{49}$ No experimento todos os tratamentos tiveram valores superiores à testemunha, diferindo estatisticamente após o engarrafamento. Esses resultados são indicativos que nos vinhos adicionados de taninos, há um desvio da absorbância do comprimento de onda característico do vermelho para o característico do amarelo.

O índice de polifenóis totais corresponde ao conjunto de todos os compostos fenólicos do vinho, absorvendo a $280 \mathrm{~nm}$ essencialmente os núcleos benzênicos, característica dos compostos fenólicos. Em princípio, cada 20 unidades de polifenóis totais representam aproximadamente $1 \mathrm{~g} . \mathrm{L}^{-1}$ de taninos. ${ }^{28}$ Os tratamentos não afetaram o teor de fenóis totais nos dois experimentos. Sabe-se que o importante para a longevidade e qualidade global de um vinho não é a extração máxima, e sim a extração seletiva destes compostos. Dal'Osto ${ }^{50}$ encontrou resultados idênticos a este trabalho, quando comparadas maceração tradicional e maceração préfermentativa a frio de vinho Syrah, cultivado em ciclo de outono-inverno em Minas Gerais.

Os tratamentos não exerceram influência quanto às antocianinas totais. Pode-se observar também que, mesmo sem tratamento estatístico, um grande decréscimo ocorreu quando comparado entre as duas avaliações (após fermentação malolática e após o engarrafamento). Este decréscimo já era esperado, em parte, já que é de senso comum que com a maturação e a estabilização dos vinhos algumas antocianinas se degradam, precipitam, resultando na formação de copigmentação estabilizado polímeros, ${ }^{51}$ a condensação tanino antocianina via etanal, ou pela formação de copolímeros entre antocianinas e quinonas de ácido caftárico. ${ }^{52}$ As antocianinas são extraídas principalmente no início da maceração, o que pode colaborar com maiores valores, já que com a maceração pré-fermentativa a frio as bagas já desengaçadas e esmagadas permanecem um período em contato com o mosto anteriormente à maceração, seguindo a vinificação tradicional posteriormente. Favre, Charamelo e GonzálezNeves $^{34}$ em seus trabalhos com a variedade Tannat, encontraram maiores valores de antocianinas para vinhos adicionados de taninos, e menores valores 
para vinhos com maceração pré-fermentativa a frio quando comparados com $o$ tratamento testemunha.

Oliva, ${ }^{53}$ afirma que os taninos enológicos adicionados proporcionam estabilidade, evitando a degradação dos compostos antociânicos. Estes resultados não foram encontrados neste trabalho, devendo-se avaliar a possibilidade de adição de quantidades maiores de taninos exógenos.

A atividade antioxidante do tratamento com uso de tanino elágico e $\mathrm{SO}_{2}$ (T4) do experimento foi significativamente superior aos demais no momento do engarrafamento.

\section{CONCLUSÕES}

A restrição do dióxido de enxofre associado ao uso de taninos elágicos aumentou a tonalidade e preservou os níveis de acidez volátil dos vinhos. Os tratamentos não exerceram influências nos resultados analíticos de índice de cor, índice de polifenóis totais, antocianinas totais e fenóis totais no momento do engarrafamento.

Embora se tratem de resultados de uma única safra, e com uma única variedade, fica evidente que é tecnicamente possível a produção de vinho tinto Merlot jovem sem a adição de $\mathrm{SO}_{2}$. Destaca-se, no entanto, que o delineamento foi estruturado usando, como modelo de estudo, uva absolutamente sãs, pressuposto enológico para que essa estratégia seja empregada. Logo, mais estudos são necessários para otimização das técnicas de vinificação empregadas e a relação destas com os parâmetros avaliados.

\section{CONTRIBUIÇÃO DOS AUTORES}

Os autores DC e GAN participaram do planejamento e condução do experimento, realizando análises e fazendo interpretação dos dados coletados. CVR e RLS definiram, orientaram e acompanharam o experimento.

\section{CONFLITO DE INTERESSE}

Os autores declaram que não há conflito de interesse.

\section{FINANCIAMENTO}

Os autores reportaram que não houve suporte e auxílio financeiro durante o desenvolvimento da pesquisa.

\section{COMO CITAR ESSE DOCUMENTO}

\section{ABNT}

CERBARO, Deisi et al. Influência da adição de taninos elágicos na qualidade de vinhos merlot da região da Campanha. Journal of bioenergy and food science, v.3, n.3, p.149-160, 2016. DOI:10.18067/jbfs.v3i3.103

\section{APA}

Cerbaro, D., Rombaldi, C., Sainz, R. \& Nobre, G. Influência da adição de taninos elágicos na qualidade de vinhos merlot da região da Campanha Journal Of Bioenergy And Food Science, 3(3) 149-160. DOI:10.18067/jbfs.v3i3.103

\section{REFERÊNCIAS BIBLIOGRÁFICAS}

1. TONIETTO, J. O conceito de denominação de origem como agente promotor de qualidade dos vinhos. Viticultura e Enologia: atualizando conceitos. Caldas: EPAMIG-FECD, p.151-163, 2002.

2. ZIRONI, R.; COMUZZO, P.; TAT, L.; ACOBIOLA, S. Trategie per la riduzione dell' SO2 nelle primi fasi della vinificazione. Infowine - Rivista Internet di Viticoltura ed Enologia, v. 12, n.2, 2009.

3. BAUTISTA-ORTÍN, A.; FERNÁNDEZ-FERNÁNDEZ, J.; LÓPEZROCA, J.; GÓMEZ-PLAZA, E. Wine-making of high coloured wines: extended pomace contact and run-off of juice prior to fermentation. Food Science and Technology International, v. 10, n. 5, p. 287-295, 2004.

4. OBRADOVIC, D.; SCHULZ, M.; OATEY, M. Adición de taninos naturales de La uva para realizar La calidad de lós vinos tintos. Revista enología. N¹1. Año II Diciembre 2005-Enero 2006.

5. GUZMÁN, F. CHARAMELO, D. GONZÁLEZ-NEVES, G. Empleo de taninos enológicos y maceración prefermentativa en frío en uma experiencia de elaboración de vinos tintos Tannat. Agrociencia Uruguay - Volumen 17 1:65-73 - enero/junio 2013.

6. PEÑA-NEIRA, A.; FREY, M.; CADAHIA, E.; FERNÁNDEZ DE SIMÓN, B.; GARCIAVALLEJO, M.C.; LOYOLA, E. Caracterización de taninos enológicos disponibles em El mercado chileno, y SUS efectos sobre um vino del cv. Merlot durante su crianza em barricas. In: CONGRÈS MONDIAL DE LA VIGNE ET DU VIN, 24, Paris, 2000. Annales, Paris: OIV.

7. LEMPEREUR, V. et al. Groupe national de travail sur lês tanins oenologiques: premiers résultats. Revue Française d'Oenologie, Paris, n.196, p.23-29, 2002.

8. GALIOTTI, H. Los taninos enológicos: revisión. Revista Enologia, v. 4, n. 1, p, 38-40, 2007.

9. MANFROI, V. RIZZON, L.A. GUERRA, C.C. FIALHO, F.B. DALL'AGNOL, I. FERRI, V.C. ROMBALDI, C.V. Influência de taninos enológicos em diferentes dosagens e épocas 
distintas de aplicação nas características físico-químicas do vinho Cabernet Sauvignon. Ciência e Tecnologia de Alimentos, v.30, n.Supl.1, p.127-135, 2010. DOI 10.1590/S0101-20612010000500020

10. VIVAS, N. Les tanins oenologiques, d'hier à aujourd'hui: une révolution discrète que nous devons assimilier dans les pratiques de chais. Revue des Oenologues, n. 98, p. 11-14, 2001.

11. ZAMORA F. Elaboración y crianza Del vino tinto: aspectos científicos y prácticos. Madrid: Mundi prensa. 225 p. 2003.

12. BRUNETON, J. Elementos de fitoquímica y de farmacognosia. Madrid: Ed. Acribia, 1991.

13. VIVAS, N.; LAGUERRE, M.; GLORIES, Y.; BOURGEOIS, G.; VITRY, C. Structures simulation of two ellagitannins from Quercus súber L. Phytochemistry, v.39,n.5, 11931199, 1995.

14. RIBÉREAU-GAYON, P.; GLORIES, Y.; MAUJEAN, A.; DUBOURDIEU, D. Phenolic Compounds. In: Handbook of enology. 2nd ed, West Sussex: John Wiley, 2002. v. 2: The chemistry of wine, stabilization and treatments, chap. 6,p. 141-203.

15. PIRIE, A.J.G.; MULLINS, M.G. Interrelationships of sugars, anthocyanins, total phenols and dry weight in the skin of grape berries during ripening. American Journal of Enology and Viticulture, v.28, p.204-209, 1977.

16. MANFROI, V.; COSTA, G. P; GUERRA, C. C.; ZANUS, M. C.; FIALHO, F. B.; ROMBALDI, C. V. Aplicação de taninos enológicos na elaboração de vinho Cabernet Sauvignon e seus efeitos sobre a qualidade sensorial. Ciência Rural, v.40, n.1, p.175-181, 2009.

17. ALVAREZ, J.M. Tanino: La revolución enológica, mito o realidad. Revista enología. n.2, Año IV Maio-Junio, 2007.

18. ORGANIZAÇÃO MUNDIAL DA UVA E DO VINHO (OIV). Resolução Oeno 12/2002. Disponível em: <http://www.oiv.int> Acesso em: 12 nov 2013.

19. MOUTOUNET, M. et al. Analyse de la composition de tanins oenologiques. Revue Française d'Oenologie, n.208, p.22-27, 2004.

20. CRESPY, A. Tanins de pépins de raisin: possibilités de stabilisation de lacouleur, de protection contrel'oxydation et d'amélioration de latenue enbouchesurles vins rouges et rosés. Revue Française d'Oenologie,v.195, p.23-29, 2002.

21. RODRIGUEZ, P.R. Utilización de taninos enológicos y virutas de roble para mejorar y estabilizar el color de los vinos tintos. Trabajo fin de Carrera. Universidade de Murcia, Departamento de tecnologia de alimentos, nutrição e bromatologia. 2006.
22. VIVAS, N. Composition et propriétés des préparation commerciales de tanins à usage oenologique. Revue des Oenologues.,v.23, p.15-21, 1997.

23. FULCRAND, H.; SALAS, E.; CHEYNIER, V. Phenolic Reactions during Winemaking and Agin. American journal of Enology and Viticulture, v.57, n.3, p. 289-297, 2006.

24. PARDO, F. Incidencia de la adición de distintos taninos enológicos en el color de los vinos tintos de Monastrell. Alimentación, equipos y tecnología, v.20, p.87-92, 2001

25. ROUSSEAU, J.; DELTEIL, D. Présentation d'une méthode $d^{\prime}$ analyse sensorielle des raisins. Principe, méthode et grille d'interprétation. Revue française d'CEnologie, v. 183, p.10-13, 2000.

26. RIZZON, L. A.; MANFROI, V.; MENEGUZZO, J. Planejamento e Instalação de uma Cantina para Elaboração de Vinho Tinto. Documento no 38. Bento Gonçalves: EMBRAPA Uva e Vinho, 2003.

27. DINIZ, B.C.R; OLIVEIRA, V.S.; ARAÚJO, A.J. B.; TRICHES, W.S.; MARTINS, A.M.; ALVES, L.A.; PEREIRA, G.E. Características físico-químicas de vinhos Merlot elaborados no Nordeste do Brasil. In: XII CONGRESSO LATINOAMERICANO DE VITICULTURA Y ENOLOGÍA. Anais... Montevideo, Uruguai, Nov. 2009.

29. RIZZON, L.A. Metodologia para análise de vinhos. Brasília, DF: Embrapa Informação Tecnológica, 120 p. 2010.

30. RIBÉREAU-GAYON, PASCAL et al. Tratado de enologia.1‥ Ed. - Hemisferio Sur, 2003. 784 p.

31. SINGLETON, V.L.; ROSSI, J.A.Jr. Colorimetry of total phenolics with phosphomolybdic phosphotungstic acid reagents. American Journal of Enology and Viticulture, v. 16, n. 3, p. 144-158, 1965.

32. LEES, D.H.; FRANCIS, F.J. Standardization of pigment analyses in cranberries. Horticultural Science, v.7, p.8384, 1972.

33. BRAND-WILLIAMS W.; CUVELIER, M. E.; BERSET, C. Use of a free radical method to evaluate antioxidant activity. Lebensmittel-Wissenschaftund Technologie, v.28, n.1, p.2530, 1995. DOI 10.1016/S0023-6438(95)80008-5

34. FAVRE, G. CHARAMELO, D. GONZÁLEZ-NEVES, G. Empleo de taninos enológicos y maceración pré fermentativa em frio en uma experiencia de elaboración de vinos tintos Tannat. Agrociencia Uruguay - Volumen 17 1:65-73 - enero/junio 2013. Prensa, 2000. 784p.

35. MIELE, A; RIZZON, L. A.; MANDELLI, F. Manejo do dossel vegetativo da videira e seu efeito na composição do vinho Merlot. Pesquisa Agropecuária Brasileira. Brasília, v.44, n.5, p.463-470, 2009. DOI 10.1590/s0100204X2009000500005 
36. RIZZON, L.A.; MIELE, A. Características analíticas de vinhos Merlot da Serra Gaúcha. Ciência Rural. v.39, n.6, p.19131916, 2009. DOI 10.1590/S0103-84782009005000109

37. RIZZON, L.A.; ZANUS, M.C.; MIELE, A. Evolução da acidez durante a vinificação de uvas tintas de três regiões vitícolas do Rio Grande do Sul. Ciência e Tecnologia de Alimentos, Campinas, v. 18, n. 2, p. 179-183, 1998. DOI 10.1590/S0101-20611998000200007

38. BRASIL. MINISTÉRIO DA AGRICULTURA, PECUÁRIA E ABASTECIMENTO. Lei BRASIL. Portaria 229, de 25 de outubro de 1988. DOU 31/10/1988. Aprova a norma referente à complementação dos padrões de identidade e qualidade do vinho. União Brasileira de Vitivinicultura (Uvibra). Disponível em: <http://www.uvibra.com.br/legislacao_portaria229.htm>. Acesso em: 02 Jan. 2014.

39.RODRÍGUEZ, P.R. Utilización de taninos enológicos y virutas de roble para mejorar y estabilizar el color de los vinos tintos. 2006. 144f. Dissertação (Mestrado em tecnologia de alimentos, nutrição e bromatologia) Universidade de Murcia, Espinardo, Espanha.

40. TAYLOR, S. L.; et al. Sulfites in foods: uses, analytical methods, residues, fate, exposure, assessment, metabolism, toxicity, and hypersensitivity. Advances in Food Research, v.30, p.1-76, 1986. DOI 10.1016/S00652628(08)60347-X

41. LECLERCQ, C.; MOLINARO, M. G.; PICCINELLI, R.; BALDINI, M.; ARCELLA, D.; STACCHINI, P. Dietary intake exposure to sulphites in Italy- analytical determination of sulphite-containing foods and their combination into standard meals for adults and children. Food Additives and Contaminants, v.17, n.12, p.979-989, 2000. DOI 10.1080/02652030010014402

42. RIBERA, D.; JONKER, D.; NARBONNE, J. F.; O'BRIEN, J.; ANTIGNAC, E. Absence of adverse effects of sodium metabisulphite in manufacture biscuits: results of subacute (28-days) and subchrionic (85-days) feeding studies in rats. Food Additives and Contaminants, v.18, n.2, p.103-114, 2001. DOI 10.1080/02652030010009174

43. FLANZY, Claude. Vinificación de los vinos de velo: vino Jaune, Jerez y otros. In. Fundamentos de Vinificación. Ediciones Mundi-Prensa. 2003. p. 531 - 539.

44. AZEVÊDO, L.C. REIS, M.M. SILVA, L.A. ANDRADE, J.A. efeito da presença e concentração de compostos carbonílicos na qualidade de vinhos. Química Nova, v.30, n.8, p.1968-1975, 2007. DOI 10.1590/S0100-40422007000800032

45. MOREIRA, N., MENDES, F., PEREIRA, O., GUEDES DE PINHO, P., HOGG, T., VASCONCELOS, I. Volatile sulphur compounds in wines related to yeast metabolism and nitrogen composition of grape musts. Analytica Chimica Acta, v.458, p.157-167, 2002. DOI 10.1016/S00032670(01)01618-X

46. MOREIRA, N., GUEDES DE PINHO, P., SANTOS, C. AND VASCONCELOS, I. Relationship between nitrogen content in grapes and volatiles, namely heavy sulphur compounds, in wines. Food Chemistry, v.126, p.15991607. 2011. DOI 10.1016/j.foodchem.2010.12.030

47. CÔRTE-REAL, D.C.C. Efeitos da maceração préfermentativa a frio e da aplicação de taninos enológicos na vinificação de tintos. 2009. 69f. Dissertação (Mestre em Viticultura e Enologia) - Instituto Superior de Agronomia, Universidade Técnica de Lisboa.

48. ZOCCHE, R.G.S. Potencial enológico de uvas Tannat, Cabernet Sauvignon e Merlot produzidas no minicípio de Bagé - RS. Pelotas, 2009, 113p. Tese (Doutorado em Ciências) - Universidade Federal de Pelotas, Pelotas.

49. RIBÉREAU-GAYON, P., GLORIES, Y., MAUJEAN, A., \& DUBOURDIEU, D. Handbook of Enology, Volume 2, The Chemistry of Wine Stabilization and Treatments. 2 nd Ed. John Wiley and Sons, Ltd. 141-205, 2006.

50. DAL'OSTO, M.C. Emprego de maceração a frio na extração e estabilização de compostos fenólicos em vinhos de Syrah cultivada em ciclo de outono-inverno. 2012. 92p. Dissertação (Mestre em Ciências) - Escola Superior de Agricultura "Luiz de Queiroz", Piracicaba, São Paulo.

51. ALONSO, E.; ESTRELLA, M.I.Y.; REVILLA, E. Los complexos polifenólicos em La elaboración y envejecimiento Del vino. Alimentación, Equipos y Tecnología, v.9-10, p.163-168, 1986.

52. CHEYNIER, V., MOUTOUNET, M.Y. SARNI MANCHADO, P. Los compuestos fenólicos. En: Enología: Fundamentos científicos y tecnológicos, Flancy, C. Ed., AMV Ediciones, 2000. pp. 114-136.

53. OLIVA, J., AZORÍN, P., CÁMARA, M. A. Y BARBA, A. Incidencia de La adición de distintos tipos de taninos enológicos em el color de vinos tintos Monastrell. Alimentación, Equipos y Tecnología, 156,87-92, 2001. 